\title{
Trayectoria de docentes que aprobaron el examen de selección para la carrera docente en el sureste de México
}

\author{
Preparation trajectory of teachers who approved the selection \\ examination for obtaining a teaching position in Southern Mexico
}

Carreira de professores aprovados em exame de seleção para a carreira docente no sudeste do México

Edith J. Cisneros-Cohernour Universidad Autónoma de Yucatán, México ecohernour@gmail.com https://orcid.org/0000-0003-2319-1519

J. Gabriel Domínguez Castillo Universidad Autónoma de Yucatán, México jg.dominguez@correo.uady.mx https://orcid.org/0000-0002-2897-913X

Pedro J. Canto Herrera Universidad Autónoma de Yucatán, México canto1962@gmail.com https://orcid.org/0000-0001-5428-8343

\section{Resumen}

Este presente estudio compara las diferencias de formación entre profesores que se graduaron de docencia o pedagogía en escuelas normales y aquellos que lo hicieron en distintos campos del conocimiento en otras instituciones de educación superior. Todos los participantes aplicaron al examen para obtener un puesto para trabajar en las escuelas públicas mexicanas. El objetivo del estudio fue determinar si las características y la trayectoria profesional de los profesores que aprobaron el examen del Sistema Profesional Docente son diferentes a las de los profesores que consiguieron su título en otras instituciones públicas o privadas. La metodología del estudio incluyó lo siguiente: (1) 


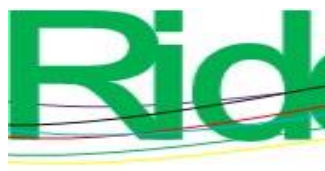

metaanálisis de las bases de datos del Centro de Evaluación Educativa de la Secretaría de Educación del Gobierno del Estado de Yucatán, y (2) una encuesta a 30 de los 43 maestros que participaron en la primera etapa del estudio. Con esta encuesta se procuró conocer las opciones de desarrollo profesional de los profesores después de tres años de aprobar el examen de docentes profesionales. Los resultados indican que hay tres grupos: aquellos que se graduaron de las escuelas normales, aquellos que se graduaron de otras instituciones de educación superior que buscan desarrollo profesional y otros que se graduaron de instituciones de educación superior que ingresaron recientemente al sistema y no tienen interés en el desarrollo profesional. Estos grupos tienen diferentes expectativas e intereses de desarrollo profesional.

Palabras clave: educación básica, formación inicial docentes, México, políticas educativas.

\section{Abstract}

This study compares the differences in training between teachers who completed a bachelor's in education o Pedagogy from the Normal Schools and those who graduated in other field rather than education from other higher education institutions. All participants applied for an examination exam to obtain a position for working in the Mexican public schools. The objective of the study was determining if the characteristics and professional trajectory of the teachers who passed the examination of the Professional Teaching System are different from those of the professors who obtained their degree in other public or private institutions. Methodology: (1) Meta-analysis of the databases of the Educational Evaluation Center of the Ministry of Education of the Government of the State of Yucatan. (2) A survey was administered to 30 of the 43 teachers who participated in the first stage of the study. The survey aimed to know teacher professional development choices after three years of approving the professional teacher examination. Results indicate that there are three groups of teachers, those who graduated from Normal Schools, those graduated from other higher education institutions seeking professional development, and other graduated from higher education institutions that recently entered the system and have no interest in professional development.

Keywords: basic education (k-12), preservice teachers, Mexico, educational policy. 


\section{Resumo}

O presente estudo compara as diferenças de formação entre professores que concluíram o magistério ou pedagogia em escolas normais e aqueles que o fizeram em diferentes áreas do conhecimento em outras instituições de ensino superior. Todos os participantes se candidataram ao exame para obter uma vaga para trabalhar em escolas públicas mexicanas. O objetivo do estudo foi verificar se as características e a trajetória profissional dos professores aprovados no concurso Sistema Profissional de Ensino são diferentes das dos professores graduados em outras instituições públicas ou privadas. A metodologia do estudo incluiu o seguinte: (1) meta-análise das bases de dados do Centro de Avaliação Educacional do Ministério da Educação do Governo do Estado de Yucatán, e (2) uma pesquisa com 30 dos 43 professores que participaram da a primeira etapa do estudo. Esta pesquisa buscou conhecer as opções de desenvolvimento profissional de professores após três anos de aprovação no exame profissional para professores. Os resultados indicam que existem três grupos: os que se formaram em escolas normais, os que se formaram em outras instituições de ensino superior em busca de desenvolvimento profissional e os que se formaram em instituições de ensino superior que ingressaram recentemente no sistema e não têm interesse no desenvolvimento profissional. Esses grupos têm expectativas e interesses diferentes para o desenvolvimento profissional.

Palavras-chave: educação básica, formação inicial de professores, México, políticas educacionais.

Fecha Recepción: Agosto 2020

Fecha Aceptación: Febrero 2021

\section{Introducción}

Los puntajes de desempeño de los estudiantes mexicanos de educación básica, principalmente de secundaria, en diferentes pruebas estandarizadas son bajos en comparación con países de primer mundo (Instituto Nacional para la Evaluación de la Educación, 2017; Márquez Jiménez, 2017). Como afirma Moreno (2016), México continúa ocupando el último lugar en cuanto a la enseñanza de matemáticas, lectura y ciencia.

Entre los factores identificados por la literatura especializada que influyen en el desempeño de los estudiantes se encuentra la formación de los profesores (Ibernón y Canto, 2013). En tal sentido, como lo mencionan varios autores (Barber, 2007; Boudersa, 2016; Hanushek, 2003; Varga, 2007), ser un maestro competente y con buen desempeño es uno de los recursos más importantes en cualquier institución educativa, ya que es el 
agente profesional y la persona más importante en el proceso de aprendizaje, pues es el encargado de ayudar a los estudiantes a mejorar sus competencias.

Por ello, es necesario profundizar en el caso de los profesores que presentan el examen del servicio profesional docente. La necesidad de este tipo de investigaciones se justifica por la escasa literatura disponible al respecto y por las diferencias de formación entre estudiantes de escuelas normales y aquellos de otras instituciones educativas públicas de México donde se aplica el referido examen para optar a cargos.

Las escuelas normales son las instituciones educativas en las que tradicionalmente se forman los docentes de educación básica en México (preescolar, primaria y secundaria). Desde su creación en 1887 con la compañía lancasteriana y su enseñanza normal, han tenido la misión de preparar a los docentes (Ducoing, 2004). En 2005, estas escuelas se incorporaron a la educación superior para procurar que, al igual que otras de nivel universitario, adoptaran una estructura similar, donde los profesores cumplen funciones no solo de docencia, sino también de tutoría, investigación y gestión. Además de las escuelas normales, las universidades tienen carreras de licenciatura en Pedagogía o Educación, y existen escuelas y universidades privadas que también preparan docentes para estos niveles y para el nivel de bachillerato.

Tomando en consideración lo expuesto en los párrafos anteriores, en la presente indagación se planteó el siguiente objetivo:

\section{Objetivo}

Determinar si las características y trayectoria profesional de los profesores que aprobaron el examen del Sistema Profesional Docente son diferentes a las de los profesores que estudiaron su licenciatura en otras instituciones públicas o privadas.

\section{Hipótesis}

Los profesores que aprobaron el examen profesional docente y realizaron estudios de licenciatura en las escuelas normales difieren significativamente en su formación y trayectoria de aquellos que obtuvieron su licenciatura en otras instituciones educativas. 


\section{Revisión de la literatura}

En México, la profesión docente es considerada como una de las más cercanas a la sociedad, pues se reconoce la importancia de su figura para formar a las nuevas generaciones mediante un proceso de fortalecimiento de la identidad nacional (Cámara de Diputados del Congreso de la Unión, 2012). Sin embargo, también vale señalar como lo mencionan Oliveira, Gonçalvez y Melo (2004) — que en los últimos años el trabajo docente ha sido cuestionado en lo referente a su formación, la cual siempre se podrá mejorar aun cuando no se cuente con el merecido apoyo material e institucional.

Debido a su estrecha relación con la sociedad, la formación del profesorado ha evolucionado a lo largo de la historia, del mismo modo que lo han hecho conceptos como escuela, enseñanza, currículo y sistema educativo. Todos estos factores han dado lugar a perfiles profesionales para el docente, como comunicador, transmisor de conocimientos, planificador y técnico, entre otros. La formación del profesorado, por tanto, se vincula con la propia evolución de la sociedad (Debesse y Mialaret, 1980).

El desarrollo profesional es la construcción de la identidad laboral, que pretende el aumento de la satisfacción en el ejercicio del trabajo por medio de una mayor comprensión y mejora de las competencias profesionales (Medina Ravilla, 1998).

Autores como Hativa (2000) indican que las razones que tiene un profesor para mejorar y querer o no su actividad docente (su preparación disciplinaria y pedagógica, su motivación para enseñar, así como sus creencias sobre lo que es la enseñanza, el aprendizaje y el rol del alumno) son las que los motiva para procurar una óptima docencia.

En general, los estudios sobre la conformación de la identidad docente han sido amplios, por lo que varias teorías han sido desarrolladas. En particular, una de las más dominantes en cuanto al desarrollo de la identidad docente — según Rodrigues y Mogarro (2019) — es el modelo de niveles de cambio de Korthagen (2004), también conocido como modelo de la cebolla. Este se encuentra constituido por una serie de niveles que interactúan para la formación de la identidad, es decir, ambiente/contexto, comportamiento, competencias, creencias, identidad y misión.

Otros desarrollos teóricos — como el de Lave y Wenger (1991) — también retoman la interacción entre la práctica y el aprendizaje, especialmente iniciando como docentes en un rol de participación relativamente periférico. Igualmente, otros modelos ubican a los significados en el centro del desarrollo de la identidad, aunque también toman en cuenta a las imágenes, percepciones y autoconocimiento que los estudiantes desarrollan de su labor como futuros docentes (Rodrigues y Mogarro, 2019). 


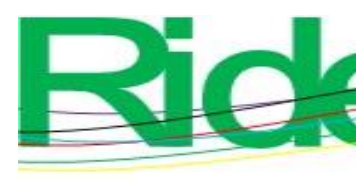

Revista Iberoamericana para la Investigación y el Desarrollo Educativo ISSN 2007 - 7467

El apoyo empírico de la relación entre formación, creencias y prácticas de las aproximaciones mencionadas anteriormente son extensas tanto en profesores con una formación tradicional como provenientes de formaciones alternativas. Por ejemplo, con respecto a los profesores de formaciones profesionales diferentes a la enseñanza, también se observa una cambio y conformación de la identidad, donde si bien se nota una continuidad en el aspecto de sus creencias, perciben más un cambio en cuestiones externas relacionadas a su ambiente de trabajo (Tigchelaar, Brouwer y Korthagen, 2008), donde se requiere una reconciliación entre su formación profesional previa y su nuevo rol como docente (Williams, 2010).

En general, las indagaciones señalan que este es un proceso dinámico que suele cambiar bidireccionalmente entre el compromiso con la enseñanza y su sol como docentes, especialmente después de la experimentación de prácticas docentes (Zhang, Clarke y Lee, 2018). Al respecto, en un interesante estudio cualitativo llevado a cabo por Deng et al. (2018), se encontró que en la práctica el desarrollo de la identidad docente de los estudiantes pasa por varias etapas: entusiasmo y ansiedad al comienzo de las prácticas de enseñanza, conmoción y vergüenza inmediatamente después de que el alumno haya enseñado, enfado y perplejidad en medio de la pasantía, impotencia y soledad hacia el final de las prácticas, y culpa y arrepentimiento después de la práctica docente. Además, los participantes se enfrentan a cuatro dilemas: (1) tensiones entre la autoridad del aula y la ética del cuidado, (2) actuar como un miembro de la comunidad o un extraño, (3) trabajar como asistente de oficina o un "maestro real", y (4) pedagogías en conflicto con respecto a la enseñanza de diferentes niveles de rendimiento académico de los estudiantes. Otros estudios han obtenido resultados similares (Oruç, 2013).

Rodrigues y Mogarro (2019) recientemente realizaron una revisión sobre los estudios de la identidad docente de estudiantes a profesores. Encontraron varias recomendaciones dirigidas a la preparación inicial de profesores que pueden resumirse en los siguientes puntos: (1) los programas deben promover el desarrollo de la identidad docente a través de actividades reflexivas y procesos de discusión; (2) se deben utilizar y reflexionar tanto en experiencias positivas como negativas de las prácticas; (3) se debe tener una aproximación sistemática para realizar actividades que promuevan el autoconocimiento y la discusión centrada en experiencias genuinas, como la exploración de sus valores y creencias como docentes, y (4) expresar de manera clara la relación entre el programa de estudios y la sociedad donde la enseñanza se llevará a cabo, lo que propiciaría que el estudiante desarrolle habilidades de afrontamiento relacionadas a sus expectativas personales y del contexto. 
Al respecto, la evidencia empírica refuerza varios elementos mencionados por Rodrigues y Mogarro. Por ejemplo, los estudios señalan los efectos positivos en del desarrollo de la identidad con tener mentores, tales como el desarrollo de la confianza y su voz como docentes (Izadinia, 2016). También se ha establecido la viabilidad del abordaje de los incidentes críticos como un método de enseñanza y reflexión, elementos relevantes para la conformación de una identidad como docente en especial para aspectos relacionados con la inclusión (Valdés y Monereo, 2012). Sin embargo, otros hallazgos señalan que la identidad docente también supone aspectos negativos, como la mala percepción al pedir ayuda o recibir retroalimentación, y poca confianza en el uso de las tecnologías (Marcelo García, 2010).

En general, como se observa, el proceso de conformación de la identidad docente, su relación con el profesionalismo y las experiencias formativas son elementos inseparables, por lo que su estudio resulta invaluable para aportar más información al respecto.

\section{Las escuelas normales}

La educación superior en México está conformada por cuatro tipos de instituciones: universidades, institutos tecnológicos, escuelas normales y universidades tecnológicas. Comprende los niveles de técnico superior universitario o profesional asociado, licenciatura, especialidad, maestría y doctorado (Instituto Nacional para la Evaluación de la Educación, 2013).

Las Instituciones de Educación Superior (IES) públicas y particulares son las responsables de formar a profesionales para la actividad docente en los distintos tipos y niveles del sistema educativo nacional, en áreas como educación preescolar, educación primaria, educación secundaria, educación especial y educación física (Cruz López y Cruz López, 2008).

La formación de docentes de educación básica la regula el Estado y está a cargo de las escuelas normales. La Constitución Política de los Estados Unidos Mexicanos, en su artículo tercero, señala que "el ejecutivo federal determinará los planes y programas de estudio de la educación preescolar, primaria, secundaria y normal para toda la república" (Diario Oficial de la Federación, 2014, p. 1).

Desde 1984, las escuelas normales ofrecen formación docente a nivel de licenciatura (por decreto presidencial), la cual requiere como antecedente el bachillerato (Candelario Sereno, 2006). Sin embargo, pese al deseo de preparar a los nuevos docentes 
como investigadores y transformadores de su hacer pedagógico, no se consiguió promover su espíritu creativo por carecer de la estructura formativa en las escuelas normales.

Tras casi doscientos años de su fundación, las escuelas normales han experimentado diferentes transformaciones, algunas relacionadas con cambios curriculares (Ducoing, 2004), requisitos de acceso para las escuelas normales y, recientemente, acceso a educación superior.

Para alcanzar la calidad educativa, las reformas de educación básica y de profesionalización del magisterio representaron nuevas exigencias para las escuelas normales. Sin embargo, su incorporación a estas fue lenta y tardía, puesto que no entraron de manera simultánea a este proceso. Por ello, en abril de 1996 se dio inicio a una amplia consulta entre la comunidad normalista del país. Después de un proceso incluyente y participativo, a fines de ese mismo año, los resultados llevaron a establecer el Programa para la Transformación y el Fortalecimiento Académicos de las Escuelas Normales. Con lo anterior, empezó la modificación de los planes y programas de estudio de las diferentes licenciaturas que se ofrecen en las escuelas normales (Candelario Sereno, 2006).

Para el periodo 2000-2006, el Programa Nacional Educativo propuso una política de formación inicial continua y desarrollo profesional permanente para renovar las normales y articular los sistemas de formación profesional en coordinación con los gobiernos estatales, así como crear redes de escuelas de educación básica para promover la especialización de los académicos. Asimismo, se planteó consolidar y articular con los estados la superación profesional para fomentar el desarrollo centrado en el aprendizaje de los alumnos (Candelario-Sereno, 2006).

A partir del año 2005, las escuelas normales fueron efectivamente consideradas como instituciones de educación superior, por lo que se creó la Dirección General de Educación Superior para Profesionales de la Educación (DGESPE) con el objetivo de proponer y coordinar las políticas educativas de educación superior para las instituciones formadoras de docentes a fin de lograr óptimos niveles de calidad y cobertura, así como su integración a las necesidades de la educación básica del país (Subsecretaría de Educación Superior, 2011). Lo anterior ha significado un enorme desafío para las escuelas normales, pues sus características estructurales, lineamientos y organización no son adecuados para afrontar las particularidades que implica formar parte del nivel superior. Actualmente, en el país operan 450 escuelas normales, de las cuales 256 (58.9\%) son de sostenimiento público y las restantes son de sostenimiento particular (Cardeña, 2012). Estas escuelas ofrecen, entre otros, programas de licenciatura en educación preescolar, 
primaria, primaria intercultural bilingüe, secundaria, especial, inicial, física y artística (Subsecretaría de Educación Superior, 2011).

A partir del ciclo escolar 2012-2013 se pusieron en marcha planes de estudio para la formación de maestros y maestras que buscan mejorar la calidad de la educación. Esta reforma atiende la imperiosa necesidad de incrementar los niveles de calidad y equidad de la educación, y asume el reto de formar docentes capaces de responder a las demandas que plantea la educación básica en los tres niveles que la integran.

\section{Desarrollo profesional del profesorado}

El Diario Oficial de la Federación (2014) define el desarrollo profesional del profesor como el proceso que sigue el personal docente y personal con funciones de dirección, supervisión y asesoría técnico-pedagógica y técnico-docente para fortalecer tanto sus competencias como su capacidad para tener los desempeños profesionales que conduzcan a la obtención de los resultados esperados en las aulas y en las escuelas públicas de educación básica.

Entre las diversas iniciativas para capacitar a profesores se encuentran el Programa Emergente para Actualizar la Formación Docente, que tiene el fin de mejorar la calidad de la formación de los maestros y maestras en servicio mediante cursos, sesiones colectivas y educación a distancia. El objetivo del programa es fortalecer, en el corto plazo, los conocimientos de los maestros y maestras para que desempeñen mejor su función (Bazdresch Parada, 2005).

Además, se estableció el Programa de Carrera Magisterial, cuyo objetivo era estimular la calidad de la educación y crear un mecanismo claro para mejorar la condición profesional, material y social de los maestros (Diario Oficial de la Federación, 1992).

\section{Investigaciones relacionadas con la formación y trayectoria docente Perspectiva internacional}

Los estudios sobre la formación y trayectoria de los docentes son abundantes, algunos de los cuales establecen diferencias entre aquellos educadores que tuvieron una formación en una escuela para educadores (como las normales o su equivalente) y aquellos que tuvieron una formación alternativa. Certificaciones alternativas para la enseñanza es un término que engloba varios tipos de programas y políticas orientadas a incluir como docentes a profesionales que no cursaron un programa de pregrado especializado en la docencia (Ludlow, 2013; Zeichner y Schulte, 2001). 
Algunos de estos programas — como mencionan Zeichner y Schulte (2001) fijan calificaciones mínimas para acreditar la incorporación de los profesionales al sistema de enseñanza. En general, se ha encontrado que los programas de certificación están asociados a docentes con edades mayores en comparación con egresados de programas de docencia tradicionales (Baeten y Meeus, 2016; Kee, 2012; Wayman, Foster, Mantle-Bromley y Wilson, 2003; Zeichner y Schulte, 2001), y suelen incluir una mayor proporción de docentes hombres que los programas tradicionales de docencia (Kee, 2012; Wayman et al., 2003). Usualmente se encuentran licenciados en otras áreas, como pueden ser las matemáticas o las ciencias en general (Kee, 2012; Unruh y Holt, 2010; Wayman et al., 2003), lo que repercute en sentirse más preparados que los docentes tradicionales, al menos en cuestiones de contenido (Kee, 2012).

Estos programas de capacitación no suelen tener el rigor académico de los especializados en la docencia de pregrado (Zeichner y Schulte, 2001). Esto posiblemente causa que los profesores noveles egresados de programas no tradicionales desarrollen un estrés generado por el desconocimiento, por lo que pueden percibirse como menos preparados para el desarrollo de varios elementos educativos, como la aplicación de técnicas de enseñanza, el desarrollo del currículo y el manejo del salón de clases (Kee, 2012; Linek, Sampson, Haas, Sadler y Moore, 2012).

Por ello, en general se recomienda un periodo de preparación para profesores egresados de profesiones no dedicadas a la docencia (Baeten y Meeus, 2016; Tigchelaar, Brouwer y Vermunt, 2010). Por ejemplo, en otros países se sugiere la necesidad de contar con mecanismos de mentorías para estos profesores (Baeten y Meeus, 2016; Fox y Peters, 2013; O’Connor, Malow y Bisland, 2011), especialmente si lo que se desea es el desarrollo de un liderazgo docente (Muijs, Chapman y Armstrong, 2013) o la autoeficacia (Fox y Peters, 2013), lo cual se puede conseguir con determinados cursos especializados (Tigchelaar et al., 2010).

Quizás por ello algunos estudios señalan que los profesores con formación alternativa en la enseñanza manifiestan una mayor preocupación por su desarrollo profesional (Wayman et al., 2003). Al respecto, los programas de capacitación ofertados y dirigidos a profesores con certificaciones alternativas para la docencia se enfocan de manera frecuente en la enseñanza y el manejo del grupo y ambiente escolar (Linek et al., 2012; Unruh y Holt, 2010; Wayman et al., 2003). Esto último resulta de especial importancia, pues este tipo de docentes tiene mayores posibilidades de experimentar eventos estresantes, como actos de violencia en el salón de clases en comparación con los profesores de programas tradicionales de docencia (Schonfeld y Feinman, 2012). 
Sin embargo, también vale destacar que revisiones recientes señalan que es importante que los profesores estén entrenados en el manejo del salón, pues incluso los programas tradicionales no abordan estos temas con la profundidad y práctica necesaria (Freeman, Simonsen, Briere y MacSuga-Gage, 2014), y en cambio suelen centrarse en el proceso de planeación y enseñanza (Alberto-Aimaretti, 2016). La inclusión de cursos orientados a cuestiones psicológicos parece también tener un impacto positivo en que los maestros, impedientemente de su formación inicial, se sientan más preparados para la enseñanza (Kee, 2012).

Con respecto a los profesores que tienen una formación "tradicional", es decir, que cursaron un programa orientado a la educación, no requieren entrenamiento en enseñanza, y suelen preocuparse más por el desarrollo de relaciones interpersonales con sus compañeros de trabajo (Linek et al., 2012). En México, los estudiantes de escuelas normales que tienen acercamientos reales a través de sus prácticas generan expectativas realistas, pues aprenden de forma vicaria de profesores más experimentados en el manejo de varios elementos del proceso formativo (Chapa Chapa y Flores Fahara, 2015), especialmente cuando dichas prácticas se brindan en escenarios no urbanos (Cardona Hernández, Hernández Madero y Lamas Mendoza, 2017), que permiten crear una identidad de agente cambio social (Chávez Monfil, Ortiz López y Ramos García, 2017). Los maestros y maestras normalistas también se encuentran interesados en el manejo de las TIC y en el conocimiento de ciertos aspectos de la psicología (Chacón Flores y Cardona Hernández, 2019).

En cuanto a la diferencia entre ambos grupos de profesores, si bien algunos estudios han encontrado que los egresados de otras profesiones tienen mejores puntajes en los exámenes de selección (Sass, 2015), en general se ha visto que no existen diferencias en el desempeño de los profesores egresados de programas tradicionales de docencia (Zeichner y Schulte, 2001), aunque los primeros tienen más posibilidades de renunciar a la profesión docente (Redding y Smith, 2016).

\section{Perspectiva local}

Entre las investigaciones que abordan dicha problemática en Yucatán, se puede mencionar el trabajo de Cisneros-Cohernour (2007), donde — según la perspectiva de administradores, docentes y autoridades de la Secretaría de Educación Pública - se identificaron problemas de desempeño profesional de docentes de ciencias de escuelas secundarias estatales de la ciudad de Mérida. 
Otro estudio realizado por Peña, Salazar y Cisneros-Cohernour (2007) examinó los diferentes factores que influyen en que los docentes participen activamente en actividades de desarrollo profesional. Los hallazgos demuestran evidencias de problemas de desarrollo profesional, así como de expectativas de los docentes.

Asimismo, los datos recabados en una revisión indican que una gran cantidad de investigaciones se han llevado a cabo desde un paradigma positivista, por lo que se caracterizan por su fuerte énfasis en la objetividad y en métodos cuantitativos de medición. Un clásico ejemplo de ello es la llamada investigación proceso-producto, que apoya la instrucción directa, la presentación y repetición del conocimiento y conducta deseados. De acuerdo con Dunkin (1986), la investigación proceso-producto de los años sesenta y principios de los setenta es el fundamento de la docencia en educación superior hoy en día. Otros investigadores se han centrado en identificar las características o cualidades utilizadas como descriptores de buena docencia, como se aprecia en la tabla 1:

Tabla 1. Características de buena enseñanza definidas en varias investigaciones

\begin{tabular}{|c|c|}
\hline Autor & Características de "buena docencia" \\
\hline $\begin{array}{l}\text { Feldman (1989): revisión de } 31 \text { estudios } \\
\text { en los cuales los estudiantes y el personal } \\
\text { docente especificaron las características } \\
\text { instruccionales que ellos consideraron } \\
\text { particularmente importante para la buena } \\
\text { enseñanza e instrucción efectiva. }\end{array}$ & $\begin{array}{l}\text { Percepción de los estudiantes de los } \\
\text { resultados de impacto de la instrucción, } \\
\text { estimula el interés de sus alumnos en el } \\
\text { curso, domina el contenido, está } \\
\text { disponible y dispuesto a ayudar a los } \\
\text { estudiantes, se preocupa y respeta a sus } \\
\text { alumnos, es amistoso, proporciona } \\
\text { retroalimentación de calidad y con la } \\
\text { frecuencia que requieren sus estudiantes, } \\
\text { es sensible y toma en cuenta el nivel de } \\
\text { clase y progreso de sus estudiantes, está } \\
\text { preparado, es organizado, anima a sus } \\
\text { estudiantes a hacer preguntas y a } \\
\text { participar en la discusión, está abierto a } \\
\text { opiniones de otros, cuida la claridad, } \\
\text { objetivos y requerimientos de otros. }\end{array}$ \\
\hline $\begin{array}{l}\text { Deshpande, Webb y Marks }(1970) \\
\text { definidas en orden de importancia por } 674 \\
\text { estudiantes de licenciatura y } 32 \text { profesores } \\
\text { de ingeniería. }\end{array}$ & $\begin{array}{l}\text { Motivación, empatía, estructura, dominio } \\
\text { del contenido, claridad, no sobrecarga a } \\
\text { sus estudiantes en sus tareas, utiliza } \\
\text { variados instrumentos de evaluación, } \\
\text { ayudas pedagógicas, habilidades } \\
\text { instruccionales y estilos de enseñanza. }\end{array}$ \\
\hline
\end{tabular}

Fuente: Adaptado de Developing Programs for Faculty Evaluation (pp. 32-33), por Miller, R. I. (1974). San Francisco CA: Jossey-Bass. 


\section{Metodología}

La investigación se realizó con base en un paradigma cuantitativo, de tipo descriptivo, y utilizó métodos mixtos de recolección de datos. Como afirman Ary, Jacobs y Razaviech (1985), este tipo de investigación permite representar a los participantes de forma precisa, ya que recaba información actual del fenómeno o permite precisar la naturaleza de una situación tal y como existe en el momento del estudio. Para su desarrollo, la investigación se desarrolló en las siguientes etapas:

\section{Primera etapa}

En esta etapa se llevó a cabo un metaanálisis de las bases de datos del Centro de Evaluación Educativa de la Secretaría de Educación del Gobierno del Estado de Yucatán. La referida base incluye información demográfica sobre las características de los profesores con y sin estudios de licenciatura normalista que han presentado el examen del Sistema Profesional Docente en 2015.

Se tomó la integración de tres bases de datos, que en conjunto sumaban N = 18954 registros. Se aplicaron filtros estrictos para elegir solo aquellos con información suficiente para realizar el análisis. El resultado fue una base de datos de tamaño $n=43$. De estos, $30(69.8 \%)$ fueron mujeres y el resto hombres. La muestra tuvo una edad promedio de 31.53 años (D. E. 6.37 años), con el participante menor de 24 años y el mayor de 47 años.

De acuerdo con el análisis de datos demográficos, se obtuvo información de las variables señaladas en la tabla 2 : 
Tabla 2. Especificación de las variables utilizadas en el estudio

\begin{tabular}{|c|c|c|}
\hline Variable & Valores o unidades & $\begin{array}{l}\text { Nivel de } \\
\text { medición }\end{array}$ \\
\hline Sexo & Hombre o mujer & Nominal \\
\hline Edad & Años & Intervalo \\
\hline Formación & Normalista u otra formación & Nominal \\
\hline Nivel académico & $\begin{array}{l}\text { Último nivel académico: licenciatura o } \\
\text { Maestría }\end{array}$ & Ordinal \\
\hline Especialización & $\begin{array}{l}\text { Área de especialización: docencia, } \\
\text { paradocencia, otras ciencias. }\end{array}$ & Nominal \\
\hline $\begin{array}{l}\text { Promedio de } \\
\text { calificación }\end{array}$ & Promedio de calificación de la licenciatura. & Intervalo \\
\hline Tiempo de titulación & Años que tardó en graduarse & Razón \\
\hline Cursos & $\begin{array}{l}\text { Tomó o no cursos, temáticas de los } \\
\text { Cursos }\end{array}$ & Nominal \\
\hline $\begin{array}{l}\text { Resultado } \\
\text { evaluación }\end{array}$ & Idoneidad medida en los niveles A, B y C & Ordinal \\
\hline
\end{tabular}

Fuente: Elaboración propia

\section{Procedimiento}

Una vez obtenida la base de datos con la información completa, se realizaron estadísticos descriptivos para conocer y caracterizar el conjunto de variables en la muestra. Posteriormente, se analizó si existían diferencias estadísticamente significativas entre aquellos con una formación en una escuela normal, en contraste con aquellos que tuvieron una formación en otro tipo de institución. Los contrastes se realizaron de la siguiente forma: para variables nominales se utilizó la prueba Ji cuadrada; y para variables ordinales, intervalo y razón se utilizó la prueba no paramétrica U de Mann-Whitney. En ambos casos, se consideró un nivel de significancia de $\alpha=.05$. Posteriormente, se analizó de forma multivariada la relación entre estas variables a partir de un análisis de correspondencias múltiples (Greenacre, 2017). Los resultados fueron representados gráficamente e interpretados de forma descriptiva.

\section{Segunda etapa}

En esta segunda etapa se trabajó con un grupo de enfoque de 12 docentes para adaptar el cuestionario de Castro (2011). Una vez adaptado el instrumento, fue revisado por personal de la Secretaría de Educación del Gobierno del Estado involucrada en formación de docentes de educación básica. En total, se realizaron tres entrevistas para mejorar la adaptación del instrumento y conocer con mayor claridad sus percepciones acerca de las diferencias entre docentes con diferentes trayectorias profesionales. 


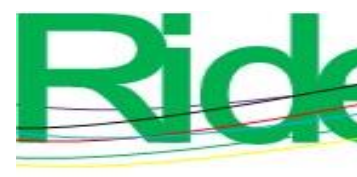

Revista Iberoamericana para la Investigación y el Desarrollo Educativo ISSN 2007 - 7467

Con base en el análisis de estas entrevistas se obtuvo la versión final del cuestionario, que se aplicó a 30 de los 43 profesores que participaron en la primera etapa del estudio con la finalidad de conocer su trayectoria tres años después de haber aprobado el examen profesional docente.

\section{Participantes}

De los 30 participantes, 23 fueron mujeres $(76.7 \%)$ y el resto hombres, con edades que van desde los 22 hasta los 46 años, con un promedio de edad de 31.76 años y una desviación estándar de 6.51. Asimismo, se determinó que $80 \%(\mathrm{n}=24)$ tenía base, mientras el resto contrato. Igualmente, $6.7 \%$ laboraba en el nivel preescolar, $53.3 \%$ en el nivel primaria, y $40.0 \%$ en el nivel secundaria. Además, $73.3 \%$ de los participantes estaba en un centro de trabajo ubicado en una zona rural, y el resto en la urbana.

\section{Instrumento}

El cuestionario incluyó aspectos relacionados con datos generales de los participantes: su formación académica, actualización académica, trayectoria profesional, situación laboral y datos sobre su centro de trabajo. Fue aplicado en su modalidad de lápiz y papel, y fue presentado a los participantes en forma de autorreporte.

\section{Procedimiento}

Para examinar los datos primero se consideró un análisis univariado, para lo cual se describieron de forma individual las variables de interés utilizando frecuencias relativas y gráficas de barras para su representación. Al respecto, se consideró si utilizaban el servicio profesional docente, otros cursos alternativos y la cantidad de cursos realizados. También se estimó la formación, posgrado y otras formas de actualización no convencionales.

Finalmente, se tomaron en cuenta factores que propician la actualización, así como las razones para no actualizarse. Seguidamente, se realizaron análisis multivariados utilizando el análisis de correspondencias múltiples para identificar relaciones entre las variables mencionadas, lo que generó un modelo de dos dimensiones. Las puntuaciones de cada participante en las dimensiones obtenidas fueron empleadas posteriormente para realizar un análisis de conglomerado para identificar grupos entre los participantes que explicaran el comportamiento de las variables. 


\section{Resultados}

A continuación, se describen los resultados del estudio. Para su mayor comprensión, en primer lugar, se presentan los datos del análisis de la base de datos, seguidos de los resultados del grupo de enfoque a los profesores que aprobaron el Sistema Profesional Docente.

\section{Resultados de la primera etapa}

Los resultados del análisis de la base de datos señalan que, del total de participantes, prácticamente $70 \%$ de la muestra seleccionada provenía de una escuela normal, mientras que $30 \%$ de otra formación. Es decir, 7 de cada 10 sustentantes fueron normalistas. Con respecto a su formación, la mayoría tuvo un nivel académico de licenciatura, y solo $14 \%$ tenía un posgrado (maestría). De hecho, la mayoría se especializaba en ciencias de la educación/docencia, seguido de paradocencia (p. ej., psicología, educación especial) y menos de $10 \%$ se especializaba en otras ciencias.

En cuanto a la distribución de las calificaciones de licenciatura obtenidas, una gran proporción (62\%) obtuvo calificaciones superiores a 8, pero inferiores a 9.5, y fue poco común conseguir calificaciones menores a 7.5 (figura 1).

Figura 1. Calificaciones de los sustentantes

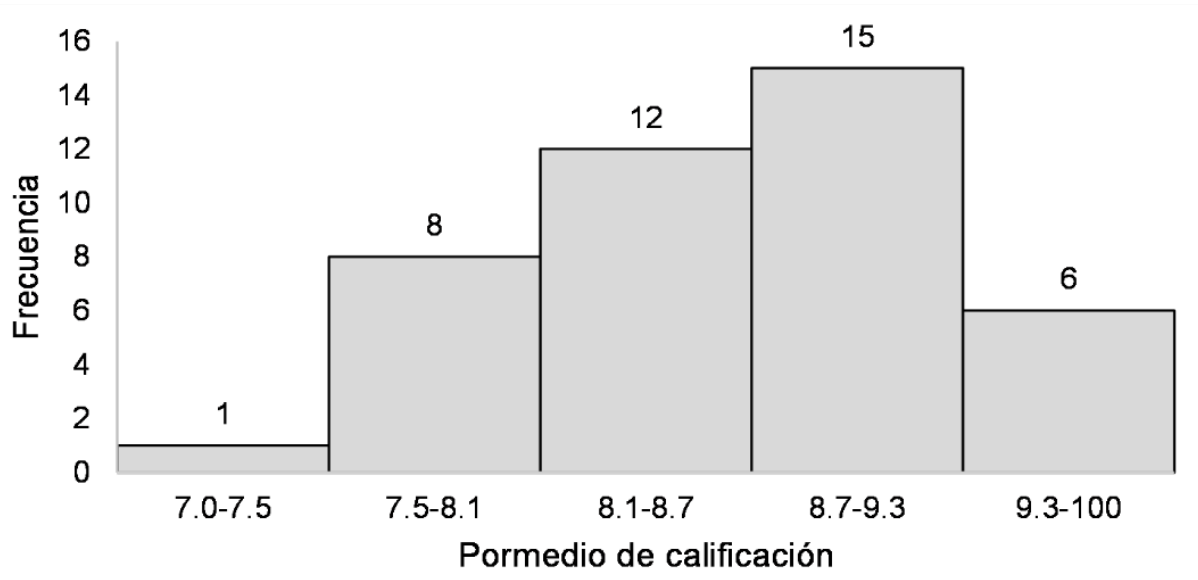

Fuente: Elaboración propia

Otro aspecto importante considerado en el instrumento fue la capacitación que los sustentantes recibieron previo al examen. Los resultados señalan que 6 de cada 10 de ellos habían cursado previamente algún diplomado o curso. En tal sentido, la mayoría de los cursos corresponde a aquellos dedicados a procesos de enseñanza-aprendizaje o desarrollo humano (20.9\% cada uno), seguido de modelos educativos (RIEB, PEP) 
(14\%), después otros (9\%), TIC (7\%) y con menor porcentaje aparecen quienes tuvieron formación en dirección o administración educativa (4.7\%).

Finalmente, se consideró el nivel que obtuvieron los participantes en la evaluación realizada para el concurso de oposición. Los resultados se aprecian en la figura 2, donde se observa que un porcentaje elevado obtuvo el nivel C, seguido por el B y el A.

Figura 2. Nivel obtenido por los participantes en la evaluación de oposición

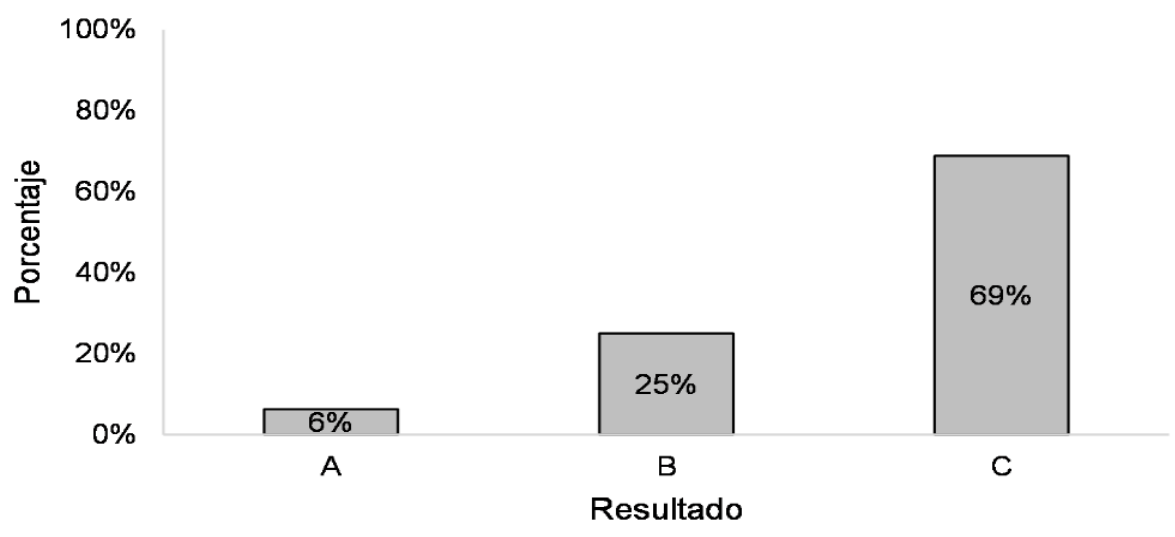

Fuente: Elaboración propia

Asimismo, se contrastó la formación de los sustentantes en relación con las variables descritas por medio de pruebas de comparación de grupos. Como se aprecia en la tabla 3, la única variable que mostró diferencias estadísticamente significativas fue la especialización de los participantes. Es decir, ni el grado académico, edad, años hasta titularse, calificación, idoneidad o cursos tomados presentaron diferencias entre aquellos que fueron formados en una escuela normalista, en contraste con los participantes formados en otro tipo de escuela. 
Tabla 3. Comparación de variables según formación de los sustentantes

\begin{tabular}{|l|c|c|c|c|}
\hline Variable $^{\dagger}$ & Normalistas & Otra formación & Estadístico & Sig. \\
\hline Grado académico & & & .032 & .858 \\
\hline Licenciatura & $86.7 \%$ & $84.6 \%$ & & \\
\hline Maestría & $13.3 \%$ & $15.4 \%$ & & \\
\hline Especialización & & & 7.494 & .024 \\
\hline Educación básica & $83.3 \%$ & $61.5 \%$ & & \\
\hline Paradocencia & $16.7 \%$ & $15.4 \%$ & & \\
\hline Otras ciencias & $0.0 \%$ & $23.1 \%$ & & \\
\hline Edad & $31.56( \pm 6.14)$ & $31.46( \pm 7.13)$ & 187.0 & .832 \\
\hline Años hasta titularse & 0 & 1 & 128.0 & .274 \\
\hline Calificación & $8.65( \pm .69)$ & $8.77( \pm .52)$ & 167.5 & .727 \\
\hline Idoneidad (B y A) & $30.0 \%$ & $33.3 \%$ & 115.0 & .811 \\
\hline Cursos de formación & $60.0 \%$ & $61.5 \%$ & .009 & .925 \\
\hline Modelos educativos & $10.0 \%$ & $23.1 \%$ & 1.292 & .256 \\
\hline Desarrollo humano & $20.0 \%$ & $23.1 \%$ & .052 & .820 \\
\hline TIC & $6.7 \%$ & $7.7 \%$ & .015 & .903 \\
\hline Dirección y administración & $6.7 \%$ & $0.0 \%$ & .909 & .340 \\
\hline Enseñanza-aprendizaje & $23.3 \%$ & $15.4 \%$ & .346 & .556 \\
\hline Otros & $10.0 \%$ & $7.7 \%$ & .057 & .811 \\
\hline
\end{tabular}

$\dagger$ Se reportan porcentajes para las variables nominales, y se reporta como estadístico la

$$
\chi^{2}
$$

Fuente: Elaboración propia

Como se observa en la tabla 3 , en ambos grupos la licenciatura fue el grado con mayor frecuencia. El promedio de edad fue similar, cercano a los 31 años. Asimismo, ambos tardaron aproximadamente de 0 a 1 años para titularse, presentaron porcentajes de postulantes idóneos similares (aproximadamente $30 \%$ ), y tuvieron un porcentaje de cursos similares (60\% aproximadamente), sin que ninguno hubiera tomado de forma más frecuente una temática de curso que otra.

La variable especialización fue la única que presentó diferencias estadísticamente significativas. Aquellos que tuvieron una formación normalista tienen una mayor probabilidad de dedicarse a la educación básica comparados con aquellos que tienen otra formación. Según los resultados, reportan especializarse en temas de educación básica como preescolar, español, matemáticas y otras asignaturas abordadas en los diversos niveles de educación básica. En contraparte, los participantes con otra formación suelen dedicarse a diversas especializaciones, como ciencias de la educación o pedagogía, lo que les permite desenvolverse en la educación básica. De hecho, estos últimos suelen tener una formación en otra ciencia (como ciencias sociales), mientras que los normalistas no presentaron ninguna especialización diferente a la rama de la educación. Sin embargo, en 
ambos casos hubo una proporción similar de especialización en tareas propias de la paradocencia, como la educación especial o la psicología.

Finalmente, cabe recalcar que los resultados anteriores fueron realizados de forma univariada, por lo que también se consideró pertinente analizar la relación multivariada entre todas las variables. Para ello, se realizó un análisis de correspondencias múltiples entre las variables de interés, considerando adicionalmente el sexo y la edad de los participantes. El resultado fue un modelo de dos dimensiones que explica $55.5 \%$ de la varianza entre las variables. La primera dimensión engloba la relación entre los cursos, el sexo, el nivel académico y la edad, y explica $31.7 \%$ de la varianza. Por su parte, la segunda dimensión, que explica $23.8 \%$ de la varianza, engloba las variables de formación, especialidad y edad.

Figura 3. Relación multivariada entre las variables de interés

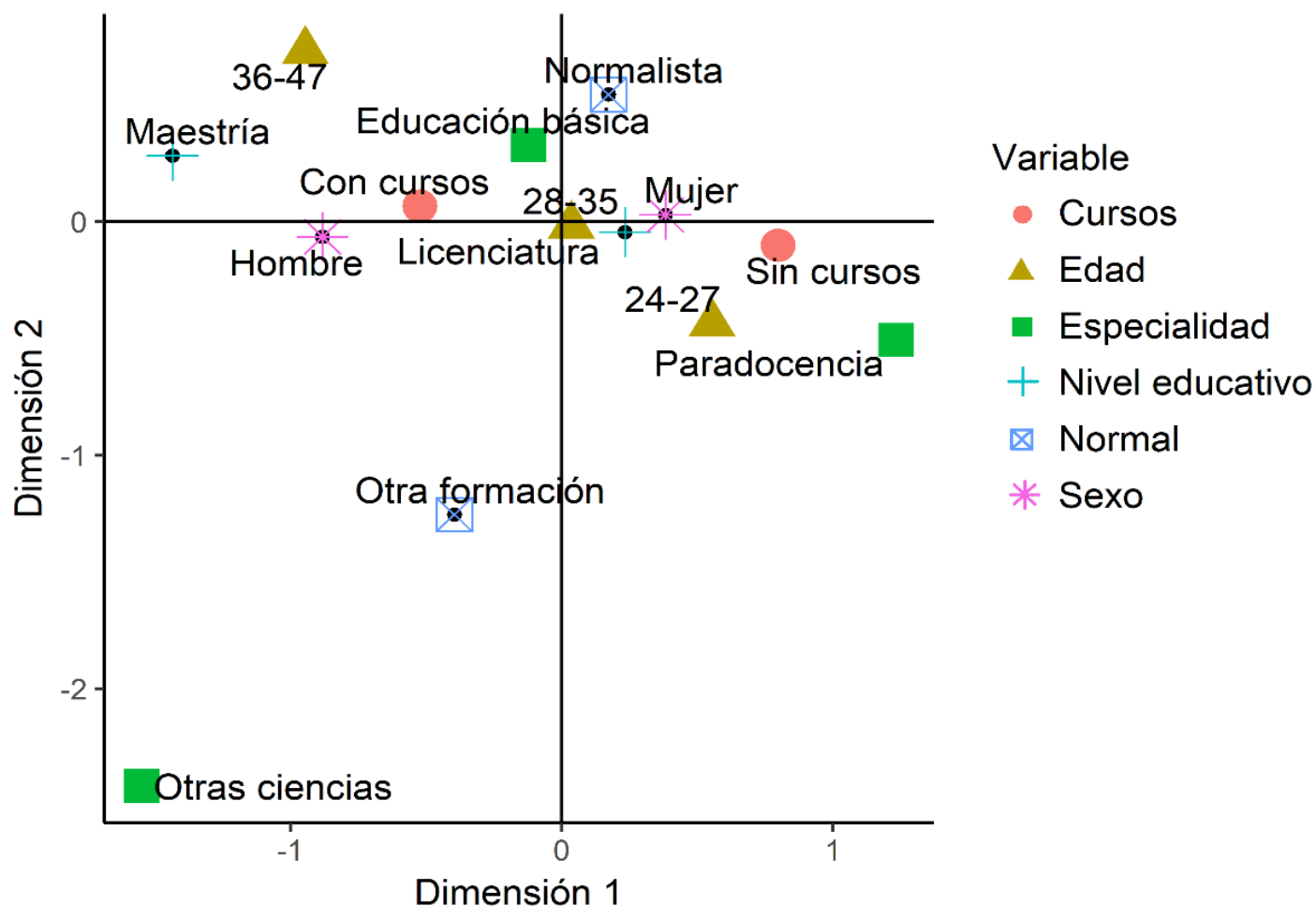

Fuente: Elaboración propia

En la figura 3 se pueden observar los siguientes patrones:

- Los paradocentes se relacionan con el hecho de no contar con cursos, y con edades jóvenes, que van de 24 a 27 años.

- Ser mujer se relaciona con tener una licenciatura, ser normalista, especializarse en la educación básica, tener una edad de 28 a 35 años y contar con cursos. Por su parte, ser hombre se relacionó con contar con cursos, tener una mayor edad (36 a 47 años) y estudiar hasta maestría. 
- Por otro lado, especializarse en otra ciencia únicamente tuvo una relación relativamente cercana con tener otra formación diferente a la normal, lo que no debe extrañar dado que la formación estuvo relacionada con la especialización.

- Finalmente, según la secuencia de las variables en el plano, parece que el tener cursos de actualización y un posgrado se asocia más con la edad que con otras variables, ya que sus patrones de alejamiento con respecto a la dimensión 1 son similares. Esto señala al tiempo o edad como la variable más relacionada con la trayectoria que cualquier otra variable estudiada.

\section{Resultados de la segunda etapa}

Como se indicó anteriormente, en esta etapa se llevó a cabo un seguimiento de 30 de los 43 profesores que participaron en la primera etapa para examinar su trayectoria luego de tres años de haber aprobado el sistema profesional docente y continuar con su práctica educativa en sus contextos. De acuerdo con los resultados, $69 \%$ de los participantes $(\mathrm{n}=20)$ estudió su formación profesional en una escuela normal, mientras que $31 \%(\mathrm{n}=9)$ lo hizo en otro tipo de institución (p. ej., universidades, tecnológicos, etc.) como se puede observar en figura 4. Cuando se analizó si existe una asociación entre la formación profesional y estudiar un posgrado, no se encontraron diferencias estadísticamente significativas entre aquellos que fueron formados en una normal en comparación de aquellos que tuvieron otra formación $\left(\chi^{2}=.019 ; \mathrm{gl}=1 ; \mathrm{p}=.625\right)$.

Figura 4. Formación profesional y de posgrado según modalidad de estudios

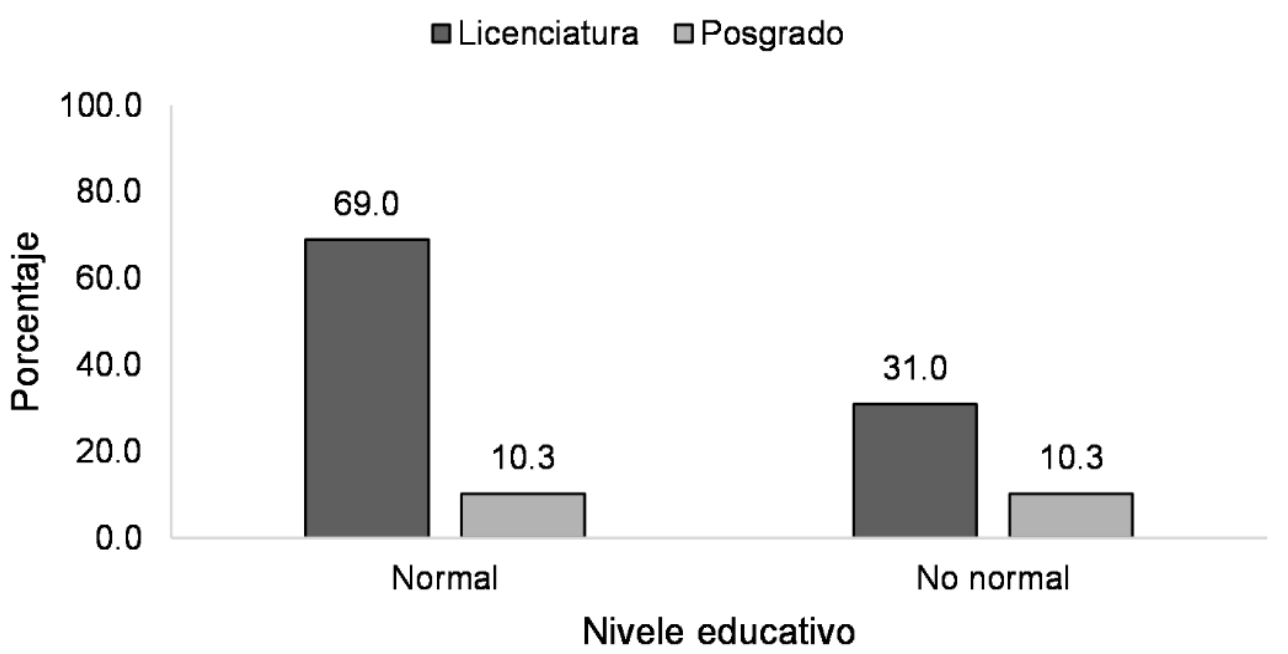

Fuente: Elaboración propia 


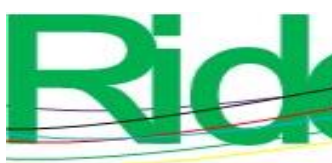

Revista Iberoamericana para la Investigación y el Desarrollo Educativo ISSN 2007 - 7467

También se exploraron los cursos que han cursado los docentes según lo ofrecido por el Consejo Nacional del Servicio Profesional Docente. Al respecto, la mayoría no ha llevado ningún curso (83.3\%). Sin embargo, existen algunos participantes que han llevado cursos en enseñanza de español (12.5\%), seguido de matemáticas (4.2\%), así como el curso nacional de integración educativa (4.2\%) o el de didáctica de los medios $(4.2 \%)$.

Del mismo modo, se exploraron las principales razones por las que se actualizan los docentes. Para 63.2 \% la principal razón es mejorar la práctica docente, seguida muy de cerca por mejorar el desarrollo académico (57.9\%) (esto de alguna forma señala el interés docente para mejorar a nivel profesional). En tercer lugar, $21.1 \%$ explicó el dominio de asignaturas, $15.8 \%$ la condición económica favorable, $10.5 \%$ cumplir con la normatividad, y $5.3 \%$ por interés y gusto personal.

Igualmente, se consideró fundamental explorar quién había recibido mayor apoyo. La mayoría opinó que fue por iniciativa propia (68.4\%); en segundo lugar, se encuentran los directivos $(21.1 \%)$, seguido de colegas $(15.8 \%)$, y en los últimos lugares se hallan autoridades educativas, supervisión escolar y "otros" (5.3\%).

Por su parte, cuando se exploraron las principales razones por las cuales no se actualizan los docentes, se obtuvo que en su mayoría se debe a la recién incorporación al sistema educativa (figura 5).

Figura 5. Razones para no actualizarse según los participantes

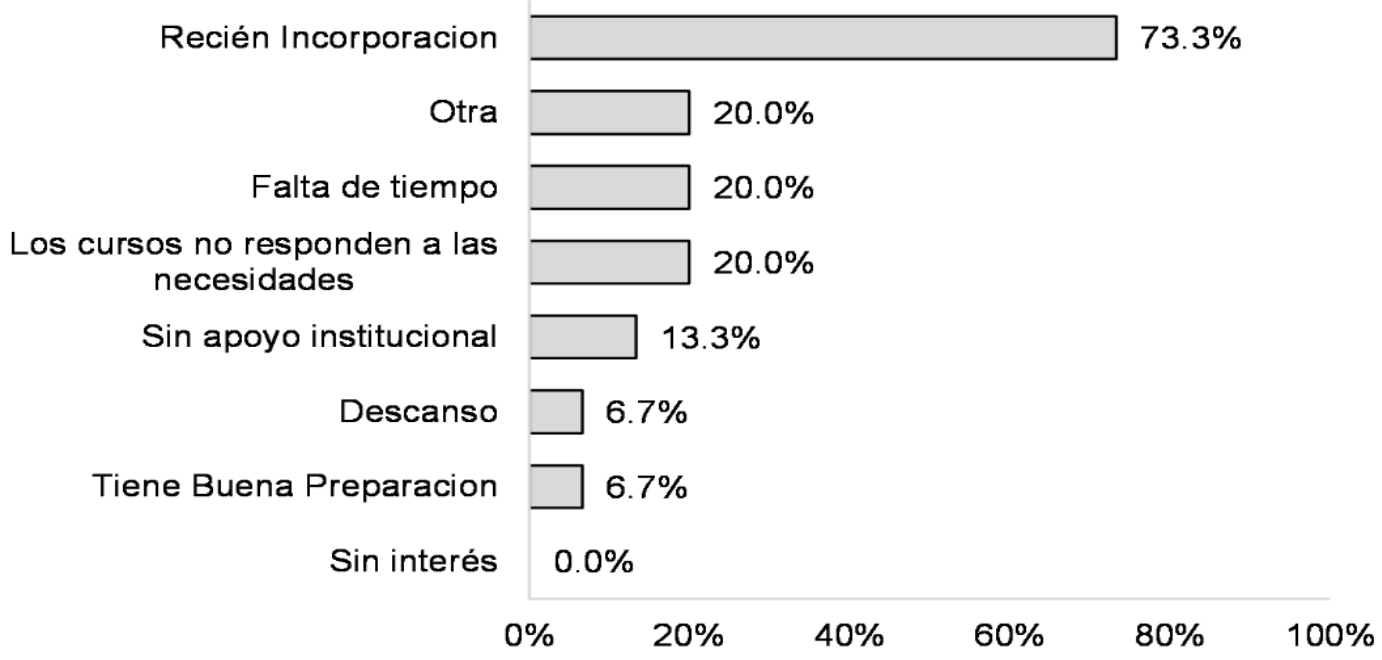

Fuente: Elaboración propia 


\section{Análisis multivariado}

Posteriormente, a partir de los descriptivos previos se decidió analizar relaciones multivariadas existentes por medio de un análisis de correspondencias múltiples que consideró las siguientes variables: formación (normal o no normal), si cuenta o no con estudios de posgrado, si se actualiza por los dos medios alternativos más frecuentes (bibliografía o internet), si ha acreditado cursos del servicio profesional docente, cuántos cursos ha realizado relacionados a su formación (cero, uno a dos, o tres a seis), si no se actualiza debido a que es de incorporación reciente, si tiene interés personal por actualizarse, si considera que debe actualizarse para mejorar su práctica docente, o si ha sido apoyado por los directivos para actualizarse.

Los análisis que consideraron estas diez variables brindaron un modelo de dos dimensiones. La primera explica $35.9 \%$ de la varianza, con una consistencia interna de $\alpha=.802$; mientras que la segunda dimensión explica $25.8 \%$ de la varianza, con una consistencia interna de $\alpha=.680$. En total, el modelo de dos dimensiones logra explicar $61.7 \%$ de la varianza de la información.

De forma general, puede observase cómo se relacionan las variables en la figura 6. Véase que, en el cuadrante superior izquierdo, se agrupan los valores de aquellas variables que no buscan actualización de ninguna forma. Por ejemplo, aquellos que no consideran que necesiten mejorar su práctica docente (PD), no usan bibliografía para actualizarse ni internet, y suelen tener poca iniciativa propia. Asimismo, en el cuadrante inferior derecho se ven aquellos valores que buscan actualización y tienen condiciones que lo favorecen. Así, por ejemplo, aquellos que buscan mejorar su práctica docente (PD) usualmente cuentan con posgrado, apoyo de directivos y usan medios alternativos para la actualización. 
Figura 6. Relación multivariada de la actualización docente, formación y motivaciones

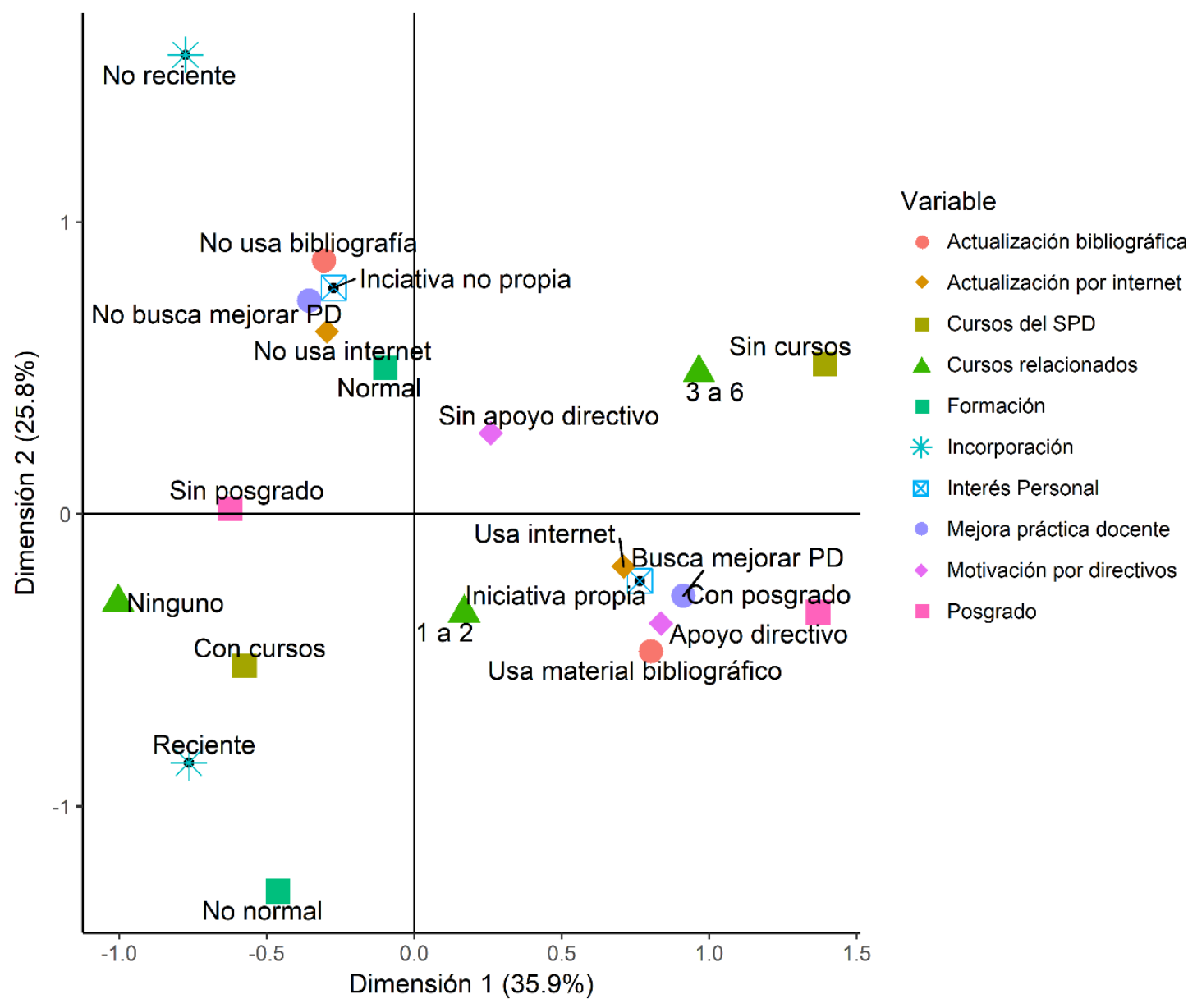

Fuente: Elaboración propia

Posteriormente, se corrieron estadísticos descriptivos de las diez variables de interés (tabla 1) para conocer las características de cada grupo. Los resultados que se presentan en la tabla 4 señalan que, en particular, el grupo 1 se encuentra conformado por normalistas, con una gran proporción de participantes sin posgrado, que en su mayoría no suelen utilizar material bibliográfico para actualizarse, y cursan programas del servicio profesional docente (SPD), pero no cursos alternativos. Además, no consideran el recién ingreso como una limitante para realizar cursos, y aunque en su mayoría tienen iniciativa propia, no suelen tener apoyo de directivos.

Por su parte, el grupo 2 está compuesto por profesionales con formación diferente a la normal, con una considerada proporción de estudios de posgrado, suelen utilizar medios alternativos para formarse (tanto libros como internet), así como cursos del SPD, y otros cursos alternativos. Sin embargo, consideran que ser de nuevo ingreso no permite la actualización de forma inicial. A pesar de ello, muestran mucha mayor iniciativa propia, apoyo de directivos y consideran importante el mejoramiento de su práctica docente. 
Tabla 4. Descriptivos de los grupos obtenidos en las variables de interés

\begin{tabular}{|c|c|c|c|c|c|c|c|}
\hline \multirow[t]{2}{*}{ Variables } & \multirow[t]{2}{*}{ Categorías } & \multicolumn{2}{|r|}{ Grupo 1} & \multicolumn{2}{|r|}{ Grupo 2} & \multicolumn{2}{|r|}{ Grupo 3} \\
\hline & & $\mathrm{N}$ & $\%$ & $\mathrm{~N}$ & $\%$ & $\mathbb{N}$ & $\%$ \\
\hline \multirow[t]{2}{*}{ Formación profesional } & Normal & 15 & $100.0 \%$ & 2 & $33.3 \%$ & 3 & $37.5 \%$ \\
\hline & No normal & 0 & $0.0 \%$ & 4 & $66.7 \%$ & 5 & $62.5 \%$ \\
\hline \multirow[t]{2}{*}{ Estudio de posgrado } & Sin posgrado & 13 & $86.7 \%$ & 2 & $33.3 \%$ & 8 & $100.0 \%$ \\
\hline & Con posgrado & 2 & $13.3 \%$ & 4 & $66.7 \%$ & 0 & $0.0 \%$ \\
\hline \multirow{2}{*}{$\begin{array}{l}\text { Se actualiza por } \\
\text { medio de material } \\
\text { bibliográfico }\end{array}$} & Sí & 5 & $35.7 \%$ & 7 & $100.0 \%$ & 0 & $0.0 \%$ \\
\hline & No & 9 & $64.3 \%$ & 0 & $0.0 \%$ & 2 & $100.0 \%$ \\
\hline \multirow[t]{2}{*}{ Actualización por internet } & Sí & 8 & $57.1 \%$ & 5 & $71.4 \%$ & 0 & $0.0 \%$ \\
\hline & No & 6 & $42.9 \%$ & 2 & $28.6 \%$ & 2 & $100.0 \%$ \\
\hline \multirow[t]{2}{*}{ Cursos acreditado del SPD } & No & 7 & $77.8 \%$ & 5 & $71.4 \%$ & 8 & $100.0 \%$ \\
\hline & Sí & 2 & $22.2 \%$ & 2 & $28.6 \%$ & 0 & $0.0 \%$ \\
\hline \multirow{3}{*}{$\begin{array}{l}\text { Cantidad de cursos } \\
\text { alternativos }\end{array}$} & Ninguno & 6 & $40.0 \%$ & 1 & $14.3 \%$ & 8 & $100.0 \%$ \\
\hline & 1 a 2 & 4 & $26.7 \%$ & 3 & $42.9 \%$ & 0 & $0.0 \%$ \\
\hline & 3 a 6 & 5 & $33.3 \%$ & 3 & $42.9 \%$ & 0 & $0.0 \%$ \\
\hline \multirow{2}{*}{$\begin{array}{l}\text { No actualización } \\
\text { por incorporación } \\
\text { reciente }\end{array}$} & Sí & 2 & $33.3 \%$ & 3 & $100.0 \%$ & 6 & $100.0 \%$ \\
\hline & No & 4 & $66.7 \%$ & 0 & $0.0 \%$ & 0 & $0.0 \%$ \\
\hline \multirow{2}{*}{$\begin{array}{l}\text { Motivación: iniciativa } \\
\text { propia }\end{array}$} & Sí & 7 & $53.8 \%$ & 6 & $100.0 \%$ & 0 & $0.0 \%$ \\
\hline & No & 6 & $46.2 \%$ & 0 & $0.0 \%$ & 2 & $100.0 \%$ \\
\hline \multirow{2}{*}{$\begin{array}{l}\text { Motivación: apoyo de } \\
\text { directivos }\end{array}$} & Sí & 2 & $15.4 \%$ & 2 & $33.3 \%$ & 0 & $0.0 \%$ \\
\hline & No & 11 & $84.6 \%$ & 4 & $66.7 \%$ & 2 & $100.0 \%$ \\
\hline \multirow{2}{*}{$\begin{array}{l}\text { Razón: mejorar práctica } \\
\text { docente }\end{array}$} & Sí & 6 & $46.2 \%$ & 6 & $100.0 \%$ & 0 & $0.0 \%$ \\
\hline & No & 7 & $53.8 \%$ & 0 & $0.0 \%$ & 2 & $100.0 \%$ \\
\hline
\end{tabular}

Fuente: Elaboración propia

Finalmente, el grupo 3 está conformado en su mayoría por profesionales con una formación en instituciones no normales, sin estudios de posgrado, que no utilizan formas alternativas de formación, así como tampoco del SPD o cursos alternativos. No se actualizan porque son de recién ingreso al sistema educativo, y aún no señalan iniciativa propia, el apoyo de directivos o la identificación de necesidad de mejorar su práctica docente.

\section{Discusión}

Los factores que pueden influir en las diferencias de formación recibida entre los distintos tipos de docentes es un tema que se viene estudiando desde hace años. En este trabajo se pudo comprobar de manera parcial la hipótesis de que los profesores que realizaron su licenciatura en las escuelas normales difieren significativamente de aquellos profesores que estudiaron su licenciatura en otras instituciones públicas o privada. Sin 
embargo, la evidencia no es totalmente contundente y deja abierto el diálogo para posibles investigaciones que profundicen en el estudio de la calidad de la formación docente.

Los resultados del estudio fueron consistentes con las investigaciones de Ludlow (2013) y Zeichner y Schulte (2001) en cuanto a que se encontraron diferencias entre los profesores que tuvieron una formación normalista y aquellos que no la tuvieron, incluso cuando estos últimos recibieron capacitación posteriormente. Esto puede deberse a que — como afirman Zeichner y Schulte (2001) y Linek et al. (2012) - los programas de capacitación para docentes que no tienen una licenciatura en educación o pedagogía tienen menos cobertura y duración, por lo que esos profesores pueden estar menos preparados para aplicar técnicas de enseñanza, desarrollar el currículo o manejar el aula. Aunque, por otro lado, aquellos cuya formación original no es en educación o pedagogía, pero en un área disciplinar, poseen un mayor dominio de contenido (Kee, 2012). Esto es importante porque un estudio realizado sobre la enseñanza de las ciencias en Yucatán encontró que los profesores de matemáticas, química, física y biología de secundaria no poseían el dominio del contenido disciplinar que enseñan en el nivel donde deben impartirlo (Cisneros-Cohernour, 2007).

Con base en los resultados, se recalca la importancia de la especialización en la formación de los profesores que laboran en las comunidades rurales como una estrategia para fortalecer la práctica docente en esos contextos. Especialmente, cuando los documentos oficiales como el PND (2019-2024) afirman que durante el periodo neoliberal el sistema de educación pública fue devastado, lo que se tradujo en la degradación de la calidad de la enseñanza en los niveles básico, medio y medio superior (Presidencia de la República, 2019).

En particular, es importante fortalecer en los profesores y profesoras en su habilitación en las tecnologías digitales, principalmente en los contextos rurales en los que laboran más de $73 \%$ de los participantes, ya que la incorporación de las tecnologías al currículo de educación básica cada día exige más de la familiaridad de los docentes con estas herramientas.

Estudios previos en contextos rurales (Domínguez Castillo, Canto Herrera, Ortega Carrillo y McCalman, 2016; Domínguez Castillo, Cisneros-Cohernour y Cab Pech, 2017; Dominguez Castillo, Cisneros Cohernour y Barberà, 2019) han documentado la importancia de la formación de profesores en las tecnologías digitales como una estrategia para fortalecer sus competencias tecnológicas, mejorar su práctica docente, el aprendizaje de sus estudiantes y favorecer la inserción en la sociedad del conocimiento y de la información de ellos y de las comunidades donde laboran. 


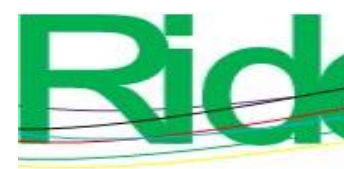

Revista Iberoamericana para la Investigación y el Desarrollo Educativo ISSN 2007 - 7467

Finalmente, se enfatiza la importancia de reforzar la profesionalización integral del personal docente a través de la capacitación como un elemento imprescindible para los docentes del nivel básico. Esta acción está contemplada en el objetivo 2.4 .2 y en la estrategia 2.4.2.1 del Plan Estatal de Desarrollo para Yucatán (2018-2024) que menciona que es importante mejorar la calidad del sistema educativo estatal y fortalecerlo en todos los niveles (Gobierno del Estado de Yucatán, 2018). Para ello, la función que desempeñan los directivos y la mejora continua que cada docente procure para su práctica docente será sumamente importante para la mejora de los aprendizajes de los estudiantes y nuestros pueblos.

\section{Conclusiones}

El trabajo realizado permitió comprobar de forma parcial la hipótesis de que los profesores que realizaron su licenciatura en las escuelas normales difieren significativamente de aquellos que estudiaron su licenciatura en otras instituciones públicas o privada. En general, se observó que no todas las variables obtuvieron diferencias estadísticamente significativas, pues únicamente el área de especialización tuvo relación con la formación de los profesores, siendo los provenientes de escuelas normales los que suelen especializarse en área vinculadas con la educación básica en sus diversos niveles, mientras que los que provienen de otras instituciones suelen especializarse en otras áreas e incluso otras ciencias. Además, ambos profesores presentan proporciones similares de especialización en áreas paradocentes, como educación especial o psicología educativa.

Sin embargo, el resto de las variables no presentó diferencias debido a que, sin considerar el área de especialización, se trata de un grupo de profesores —en su mayoría - con estudios generales de licenciatura, que cursan la misma proporción y temáticas de cursos, y cuyo tiempo de titulación (aproximadamente un año) es prácticamente igual. Si bien de forma inicial se consideró que posiblemente estas variables puedan diferenciar en ambas formaciones, lo cierto es que no lo hizo. Es posible que esto se deba a que las convocatorias tienden a exigir ciertos criterios homogéneos para la oposición y selección de los docentes.

Por su parte, si bien el tomar cursos y la temática de ellos también se consideró como una posible variable que presentara diferencias, esta no lo hizo. Nuevamente es posible que se deba a que los cursos ofrecidos a los profesores son tanto obligatorios o bien abiertos a cualquier interesado, por lo que la cantidad o temática no resulta un 
diferenciador de la formación docente. Sin embargo, los descriptivos obtenidos ponen de manifiesto una cosa importante: ciertas temáticas especialmente relevantes no presentan una prevalencia alta, como por ejemplo el uso de las TIC o los cursos especializados en habilidades directivas o de administración educativa.

Lo anterior es especialmente relevante por dos consideraciones. Por una parte, resulta apropiado ofrecer formación a los profesores con respecto al manejo de las TIC, pues el uso y penetración de estas tecnologías no solo es más frecuente en la vida cotidiana, sino que su incorporación en el currículo de la educación básica cada vez exige una mayor familiaridad con los temas. Por ejemplo, hoy en día es común que en primaria no solo se expliquen las características y bondades del correo ordinario, sino que a la par se incluye el uso del correo electrónico, por lo que resulta relevante que a los docentes se les ofrezca y exija el conocimiento en las TIC.

Los resultados obtenidos en el seguimiento de los profesores después de tres años de haber aprobado el sistema profesional docente indican, a partir de los análisis univariados y multivariados, que al menos hay cinco posibles conclusiones que se deben considerar.

Existen variables contextuales y personales que propician la actualización de los docentes, tales como el apoyo de los directivos o la identificación de una necesidad de mejorar la práctica docente que deben incluirse en futuros programas que busquen incidir en la actualización docente.

La obtención de tres grupos (normalistas, no normalistas que buscan actualización, y no normalistas de recién ingreso) señala la presencia de factores propios de cada grupo.

Por un lado, si bien los normalistas buscan formación más formal (como el servicio profesional docente), y menos otras maneras de actualización alternativa, es posible que esto se deba a que su formación inicial está plenamente relacionada y focalizada en el campo educativo y docente, lo que posiblemente repercute en considerar en menor medida la necesidad de mejorar su práctica docente.

Por otra parte, los no normalistas que buscan actualización probablemente presenten formaciones lejanas a la docencia o al menos no tan focalizadas como los normalistas, razón por la que además de la actualización formal también buscan medios alternativos para actualizarse. Es posible que esto se deba a que su formación hace que consideren necesario mejorar su práctica docente, y por tanto busquen activamente cursos y oportunidades de formación. Además, es posible también que los directivos noten esta necesidad, por lo que suelen contar con mayor apoyo para mejoramiento. 
Finalmente, los no normalista de nuevo ingreso aún no buscan la actualización, y consideran su recién ingreso como una limitante para saber qué cursar. Posiblemente su falta de relación práctica con la labor docente también explique que aún no identifican la mejora docente como una necesidad.

\section{Recomendaciones}

El estudio nos permite recomendar que posiblemente deban considerarse al menos tres programas de actualización docente por separado según los grupos identificados. Por ejemplo, el grupo de normalistas, al tener una sólida formación en docencia, posiblemente no requiera cursos relacionados con la enseñanza-aprendizaje, sino con otras áreas que deberían explorarse con mayor profundidad. Por su parte, los no normalistas requieren al menos dos planes: uno para aquellos que inician y que deben buscar sus necesidades de actualización y, por otra parte, los que ya están inmersos en el sistema educativo y que posiblemente requieran no solo formación en aspectos docentes, sino también mecanismos facilitadores (mayor apoyo de directivos y colegas, y formación alternativa en internet o en fuentes bibliográficas). Sin duda, los programas de actualización docente deben considerar la homogeneidad de estos tres grupos.

En segundo lugar, brindar cursos de habilidades directivas y de administración educativa resulta especialmente relevante si se considera que muchos profesores pueden tener la oportunidad de crecer profesional u organizacionalmente, ya sea al cubrir puestos directivos en las escuelas o bien en la misma Secretaría de Educación. Esto último pone de manifiesto la necesidad de que los docentes conozcan y cuenten con habilidades que les permitan gestionar y administrar adecuadamente los recursos de su plantel o de su futura área. Por tanto, se considera apropiado ofrecer cursos teniendo en cuenta un plan de crecimiento organizacional en los profesores.

Finalmente, los resultados también demostraron la relación que existe entre la formación y la trayectoria docente con otras variables de forma multivariada. En el análisis, si bien se obtuvo información consistente con los análisis previos (como la relación entre la especialización y la formación), también se consiguió información adicional, como el hecho de que tomar cursos o el nivel educativo (aspectos relacionados con la formación continua) se asocian más directamente con la edad de los profesores que con otra variable. Sin duda, lo anterior no es un resultado inesperado, ya que una edad mayor se vincula con el haber tenido más oportunidades de formación continua, aunque esto también refleja la necesidad de comenzar con un plan de formación desde edades tempranas en la carrera magisterial, además de considerar quizás un plan de desarrollo y 
formación continua planificado en función de la edad y el desarrollo del individuo en la organización educativa.

En resumen, el presente estudio permitió detectar diferencias (aunque únicamente en cuestión del área de especialización) en la formación de los profesores que cursaron sus estudios en escuelas normales en comparación con aquellos que estudiaron en otro tipo de instituciones. En tal sentido, la trayectoria de formación continua parece estar más influenciada por la edad de los docentes, y no tanto por la institución de procedencia.

\section{Futuras líneas de investigación}

Con base en las conclusiones, futuros estudios pueden examinar con mayor profundidad la calidad del desempeño docente de los profesores con diferentes tipos de formación, así como la medida en que las condiciones del contexto en donde desarrollan la docencia favorecen la actualización y la formación docente.

Dadas las nuevas condiciones en que se lleva a cabo la educación a distancia, a raíz de la pandemia de la covid-19, futuros estudios necesitan examinar cómo este contexto se ha visto afectado y cómo los docentes responden a las condiciones impuestas por la pandemia, así como la existencia de apoyos para docentes y estudiantes para desarrollar la docencia a distancia y los obstáculos y aprendizajes más significativos y las nuevas necesidades de formación del profesorado. 


\section{Referencias}

Alberto-Aimaretti, E. S. (2016). Formación de docentes para los niveles inicial y primario. Revista Iberoamericana de Educacion Superior, 7(19), 181-193. Doi: https://doi.org/10.35362/rie672257

Ary, D., Jacobs, L. C. and Razavieh, A. (1985). Introduction to Research in Education ( $3^{\text {th }}$ ed.). Nueva York: Holt, Rinehart and Winston.

Baeten, M. and Meeus, W. (2016). Training Second-Career Teachers: A Different Student Profile, A Different Training Approach? Educational Process: International Journal, 5(3), 173-201. Doi: https://doi.org/10.12973/edupij.2016.53.1

Barber, M. (2007). How the World's Best-performing Schools Systems Come Out on Top. Nueva York: McKinsey \& Company.

Bazdresch Parada, M. (2005). La investigación educativa: notas de un campo problemático. En Rojo, S., Mondragón, L., Arrona, M. y Zenteno, H. (eds.), Linderos. Diálogos sobre investigación educativa (pp. 177-187). Ciudad de México: Consejo Mexicano de Investigación Educativa.

Boudersa, N. (2016). The Importance of Teachers' Training Programs and Professional Development in the Algerian Educational Context: Toward Informed and Effective Teaching Practices. Orán: École Normale Superieure D’Oran.

Cámara de Diputados del Congreso de la Unión (2012). Iniciativa de decreto que reforma y adiciona diversas disposiciones de la Constitución Política de los Estados Unidos Mexicanos. Ciudad de México: Congreso de la Unión.

Candelario Sereno, T. (2006). La formación del docente y los procesos de profesionalización para construir competencias para la enseñanza. Congreso Estatal de Investigación Educativa Actualidad, Prospectivas y Retos. Guadalajara.

Cardeña, C. (2012). Reflexiones sobre la reforma curricular de la educación normal. Diálogos sobre educación. Temas actuales en investigación educativa. Vol. 3, num. 5. Julio-diciembre. Pp. 1-14. Universidad de Guadalajara. Zapopan, México.

Cardona Hernández, A., Hernández Madero, J. y Lamas Mendoza, L. C. (2017). La caracterización de la identidad profesional de los maestros en formación de un grupo de primer grado de la licenciatura en educación primaria de la IBYCENECH. Congreso Nacional de Investigación sobre Educación Normal (pp. 1-5). Mérida, Yucatán.

Castro, J. (2011). Cuestionario para docentes de secundaria en servicio. Ciudad de 
México: Instituto Nacional para la Evaluación de la Educación.

Chacón Flores, S. y Cardona Hernández, A. (2019). Identidad profesional docente en la formación inicial. Congreso Nacional de Investigación sobre Educación Normal (pp. 1-9). Playa de Rosarito, Baja California.

Chapa Chapa, M. y Flores Fahara, M. (2015). La formación inicial de profesores en las escuelas normales. IE Revista de Investigación Educativa de la REDIECH, 6(10), 28-35. Doi: https://doi.org/10.33010/ie_rie_rediech.v6i10.168

Chávez Monfil, M., Ortiz López, M. T. y Ramos García, F. B. (2017). La formación de la identidad profesional en la voz de los estudiantes normalistas. Congreso Nacional de Investigación sobre Educación Normal (pp. 1-9). Mérida, Yucatán.

Cisneros-Cohernour, E. J. (2007). La enseñanza de las Ciencias en Yucatán: Cuestiones críticas, retos y posibles soluciones. Mérida: CONACYT-UADY-Unas Letras.

Cruz López, Y. y Cruz López, A. K. (2008). La educación superior en México tendencias y desafíos. Avaliação: Revista da Avaliação da Educação Superior (Campinas), 13(2), 293-311. Doi: https://doi.org/10.1590/S1414-40772008000200004

Debesse, M. y Mialaret, G. (1980). La función docente. Barcelona: Oikos-Tau, S.A.

Deshpande, A. S., Webb, S. C., \& Marks, E. (1970). Student Perceptions of Engineering Instructor Behaviors and Their Relationships to the Evaluation of Instructors and Courses. American Educational Research Journal, 7(3), 289-305. https://doi.org/10.3102/00028312007003289

Deng, L., Zhu, G., Li, G., Xu, Z., Rutter, A. and Rivera, H. (2018). Student Teachers' Emotions, Dilemmas, and Professional Identity Formation Amid the Teaching Practicums. Asia-Pacific Education Researcher, 27(6), 441-453. Doi: https://doi.org/10.1007/s40299-018-0404-3

Diario Oficial de la Federación (1992). Acuerdo nacional para la modernizacion de la educación básica. Ciudad de México: Congreso de la Unión. Recuperado de https://www.sep.gob.mx/work/models/sep1/Resource/b490561c- 5c33-4254ad1c-aad33765928a/07104.pdf

Diario Oficial de la Federación (2014). Acuerdo número 712 por el que se emiten las reglas de operación del Programa para el Desarrollo Profesional Docente. Ciudad de México: Congreso de la Unión. Recuperado de http://dof.gob.mx/nota_detalle.php?codigo=5328399\&fecha=29/12/2013

Domíngguez Castillo, J. G., Canto Herrera, P. J., Ortega Carrillo, J. A. and McCalman, D. G. (2016). Raising the technological competence of high school science and mathematics teachers of Mexico through delivery of an online program. 


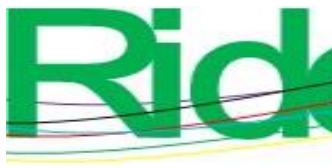

Revista Iberoamericana para la Investigación y el Desarrollo Educativo ISSN 2007 - 7467

International Journal of Technology, Policy and Management, 16(2), 163. Doi: https://doi.org/10.1504/IJTPM.2016.076317

Domínguez Castillo, J. G., Cisneros-Cohernour, E. J. y Cab Pech, V. M. (2017). Familiaridad en el uso de las tencologías digitales en comunidades vulnerables del sur de Yucatán. En Mortis Lozoya, S. V., Muñoz Arteaga, J. y Zapata González, A. (eds.), Reducción de brecha digital e inclusión educativa: Experiencias en el Norte, Centro y Sur de México (pp. 175-194). Naucalpan: Grupo Editorial Rosa María Porrúa.

Dominguez Castillo, J. G., Cisneros Cohernour, E. J. and Barberà, E. (2019). Factors influencing technology use by Mayan women in the digital age. Gender, Technology and Development, 22(3), 185-204. Doi: https://doi.org/10.1080/09718524.2018.1558862

Ducoing, P. (2004). Origen de la escuela normal superior de México. Revista Historia de la Educación Latinoamericana, 6(6), 39-56.

Dunkin, M. J. (1986). Research on teaching in higher education. In Wittroc, M. C. (ed.), Handbook of Research on Teaching (pp. 754-777). Michigan: American Educational Research Association.

Feldman, K. A. (1989). Instructional effectiveness of college teachers as judged by teachers themselves, current and former students, colleagues, administrators, and external (neutral) observers. Research in Higher Education, 30(2), 137-194. Doi: https://doi.org/10.1007/BF00992716

Fox, A. G. and Peters, M. L. (2013). First year teachers: Certification program and assigned subject on their self-efficacy. Current Issues in Education, 16(1), 1-16.

Freeman, J., Simonsen, B., Briere, D. E. and MacSuga-Gage, A. S. (2014). Pre-Service Teacher Training in Classroom Management. Teacher Education and Special Education: The Journal of the Teacher Education Division of the Council for Exceptional Children, 37(2), 106-120. Doi: https://doi.org/10.1177/0888406413507002

Gobierno del Estado de Yucatán. (2018). Plan Estatal de Desarrollo (2018-2024). Mérida: Gobierno del Estado de Yucatán. Recuperado de http://www.yucatan.gob.mx/docs/transparencia/ped/2018_2024/2019-0330_2.pdf

Greenacre, M. (2017). Correspondence Analysis in Practice ( $3^{\text {th }}$ ed.). Boca Ratón: CRC Press.

Hanushek, E. A. (2003). The Failure of Input-based Schooling Policies. The Economic 


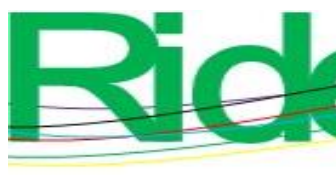

Revista Iberoamericana para la Investigación y el Desarrollo Educativo ISSN $2007-7467$

Journal, 113(485), F64-F98. Doi: https://doi.org/10.1111/1468-0297.00099

Hativa, N. (2000). Becoming a better teacher: A case of changing the pedagogical knowledge and beliefs of law professors. Instructional Science, 28, 491-523. Doi: https://doi.org/10.1023/A:1026521725494

Ibernon, F., Canto, P. J. (julio-diciembre, 2013). La formación y el desarrollo profesional del profesorado en España y Latinoamérica. Sinéctica, 41. Recuperado de http://www.sinectica.iteso.mx/articulo/?id=41_

la_formacion_y_el_desarrollo_profesional_del_profesorado_en_espana_y_latin oamerica

Instituto Nacional para la Evaluación de la Educación. (2013). Estructura y dimensión del Sistema Educativo Nacional. Ciudad de México: Instituto Nacional para la Evaluación de la Educación. Recuperado de http://www.inee.edu.mx/bie_wr/mapa_indica/2013/

Instituto Nacional para la Evaluación de la Educación (2017). México en el proyecto TALIS-PISA: un estudio exploratorio. Importancia de las escuelas, directores, docentes y estudiantes en el aprendizaje de las matemáticas. Ciudad de México: Instituto Nacional para la Evaluación de la Educación.

Izadinia, M. (2016). Preservice teachers' professional identity development and the role of mentor teachers. International Journal of Mentoring and Coaching in Education, 5(2), 127-143. Doi: https://doi.org/10.1108/IJMCE-01-2016-0004

Kee, A. N. (2012). Feelings of preparedness among alternatively certified teachers: What is the role of program features? Journal of Teacher Education, 63(1), 23-38. Doi: https://doi.org/10.1177/0022487111421933

Korthagen, F. A. J. (2004). In search of the essence of a good teacher: Towards a more holistic approach in teacher education. Teaching and Teacher Education, 20(1), 77-97. Doi: https://doi.org/10.1016/j.tate.2003.10.002

Lave, J. and Wenger, E. (1991). Situated Learning: Legitimate Peripheral Participation. Cambridge: Cambridge University Press. Doi: https://doi.org/10.1017/CBO9780511815355

Linek, W. M., Sampson, M. B., Haas, L., Sadler, D. and Moore, L. (2012). The impact of teacher preparation a study of alternative certification and traditionally prepared teachers in their first year of teaching. Issues in Teacher Education, 2(2), 67-83.

Ludlow, C. (2013). Alternative Certification Pathways: Filling a Gap? Education and Urban Society, 45(4), 440-458. Doi: https://doi.org/10.1177/0013124511413916

Marcelo García, C. (2010). La identidad docente: constantes y desafíos. Revista 


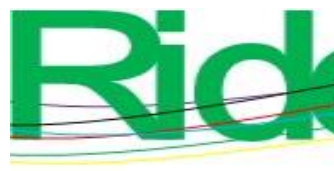

Revista Iberoamericana para la Investigación y el Desarrollo Educativo ISSN $2007-7467$

Interamericana de Investigación, Educación y Pedagogía, 2(1), 15-42.

Márquez Jiménez, A. (2017). A 15 años de PISA: resultados y polémicas. Perfiles Educativos, $39(156)$.

Doi: https://doi.org/10.22201/iisue.24486167e.2017.156.58280

Medina Ravilla, A. (1998). Organización de la formación y desarrollo profesional del docente universitario. $V$ Congreso Interuniversitario de Organización de Instituciones Educativas (pp. 697-790). Madrid.

Moreno, T. (2016, 6 de diciembre), “OCDE: México, 15 años en el último lugar de educación",El Universal, Nación, en: http://www.eluniversal.com.mx/articulo/nacion/sociedad/2016/12/6/ocdemexico-15-anos-en-el-ultimo-lugar-de-educacion (consulta: 10 de marzo de 2020).

Muijs, D., Chapman, C. and Armstrong, P. (2013). Can Early Careers Teachers Be Teacher Leaders? A Study of Second-year Trainees in the Teach First Alternative Certification Programme. Educational Management Administration and Leadership, 41(6), 767-781. Doi: https://doi.org/10.1177/1741143213494188

O’Connor, E. A., Malow, M. S. and Bisland, B. M. (2011). Mentorship and instruction received during training: Views of alternatively certified teachers. Educational Review, 63(2), 219-232. Doi: https://doi.org/10.1080/00131911.2010.537312

Oliveira, D. A., Gonçalves, G. B. y Melo, S. (2004). Cambios en la organización del trabajo docente: Consecuencias para los profesores. Revista Mexicana de Investigación Educativa, 9(20), 183-197.

Oruç, N. (2013). Early Teacher Identity Development. Procedia - Social and Behavioral Sciences, 70, 207-212. Doi: https://doi.org/10.1016/j.sbspro.2013.01.056

Peña, S., Salazar, A. y Cisneros-Cohernour, E. J. (2007). Factores que influyen en el desarrollo profesional de docentes de ciencias. En Cisneros-Cohernour, E. J. (ed.), La enseñanza de las Ciencias en Yucatán: Cuestiones críticas, retos y posibles soluciones. Mérida: Conacyt-UADY-Unas Letras.

Presidencia de la República (2019). Plan Nacional de Desarrollo (2019-2024). Ciudad de México: Gobierno de México. Recuperado de https://lopezobrador.org.mx/wpcontent/uploads/2019/05/PLAN-NACIONAL-DE-DESARROLLO-20192024.pdf

Redding, C. and Smith, T. M. (2016). Easy in, Easy out: Are Alternatively Certified Teachers Turning Over at Increased Rates? American Educational Research Journal, 53(4), 1086-1125. Doi: https://doi.org/10.3102/0002831216653206 
Rodrigues, F. y Mogarro, M. J. (2019). Student teachers' professional identity: A review of research contributions. Educational Research Review, 28(April), 100286. Doi: https://doi.org/10.1016/j.edurev.2019.100286

Sass, T. R. (2015). Licensure and worker quality: A comparison of alternative routes to teaching. Journal of Law and Economics, 58(1), 1-35. Doi: https://doi.org/10.1086/682904

Schonfeld, I. S. y Feinman, S. J. (2012). Difficulties of Alternatively Certified Teachers. Education and Urban Society, 44(3), 215-246. Doi: https://doi.org/10.1177/0013124510392570

Subsecretaría de Educación Superior (2011). Reforma curricular de educación normal. Licenciatura en Educación Primaria. Licenciatura en Educación Preescolar. Licenciatura en Educación Preescolar Intercultural Bilingüe. Ciudad de México: Subsecretaría de Educación Superior.

Tigchelaar, A., Brouwer, N. and Korthagen, F. (2008). Crossing horizons: Continuity and change during second-career teachers' entry into teaching. Teaching and Teacher Education, 24(6), 1530-1550. Doi: https://doi.org/10.1016/j.tate.2008.03.001

Tigchelaar, A., Brouwer, N. and Vermunt, J. D. (2010). Tailor-made: Towards a pedagogy for educating second-career teachers. Educational Research Review, 5(2), 164-183. Doi: https://doi.org/10.1016/j.edurev.2009.11.002

Unruh, L. and Holt, J. (2010). First-Year Teaching Experiences: Are They Different for Traditionally versus Alternatively Certified Teachers? Action in Teacher Education, 32(3), 3-14. Doi: https://doi.org/10.1080/01626620.2010.10463555

Valdés, A. M. y Monereo, C. (2012). Desafíos a la formación del docente inclusivo: la identidad profesional y su relación con los incidentes críticos. Revista Latinoamericana de Educación Inclusiva, 6(2), 193-208.

Varga, J. (2007). Kiből lesz ma tanár?. A tanári pálya választásának empirikus elemzése. [Who becomes a teacher today?. An empirical analysis of choosing the teaching profession]. Közgazdasági Szemle, 54(7-8), 609-627.

Wayman, J. C., Foster, A. M., Mantle-Bromley, C. and Wilson, C. A. (2003). A Comparison of the Professional Concerns of Traditionally Prepared and Alternatively Licensed New Teachers. The High School Journal, 86(3), 35-40. Doi: https://doi.org/10.1353/hsj.2003.0005

Williams, J. (2010). Constructing a new professional identity: Career change into teaching. Teaching and Teacher Education, 26(3), 639-647. Doi: https://doi.org/10.1016/j.tate.2009.09.016 
Zeichner, K. M. y Schulte, A. K. (2001). What we know and don't know from peerreviewed research about alternative teacher certification programs. Journal of Teacher Education, 52(4), 266-282. Doi: https://doi.org/10.1177/0022487101052004002

Zhang, Q., Clarke, A. and Lee, J. C. K. (2018). Pre-service Teachers' Professional Identity Development Within the Context of School-Based Learning to Teach: An Exploratory Study in China. Asia-Pacific Education Researcher, 27(6), 477-486. Doi: https://doi.org/10.1007/s40299-018-0408-z 


\begin{tabular}{|c|c|}
\hline Rol de Contribución & Autor (es) \\
\hline Conceptualización & Edith J. Cisneros Cohernour \\
\hline Metodología & $\begin{array}{l}\text { Edith J. Cisneros Cohernour «principal» J. Gabriel Dominguez } \\
\text { Castillo «apoyo». }\end{array}$ \\
\hline Software & Pedro J. Canto Herrera \\
\hline Validación & $\begin{array}{l}\text { Edith J. Cisneros Cohernour «principal» J. Gabriel Dominguez } \\
\text { Castillo «apoyo» y Pedro J. Canto Herrera «apoyo» }\end{array}$ \\
\hline Análisis Formal & $\begin{array}{l}\text { Edith J. Cisneros Cohernour «principal» J. Gabriel Dominguez } \\
\text { Castillo «igual» Pedro J. Canto Herrera «apoyo». }\end{array}$ \\
\hline Investigación & $\begin{array}{l}\text { Edith J. Cisneros Cohernour «principal» J. Gabriel Dominguez } \\
\text { Castillo «igual» y Pedro J. Canto Herrera «apoyo». }\end{array}$ \\
\hline Recursos & Edith J. Cisneros Cohernour \\
\hline Curación de datos & $\begin{array}{l}\text { J. Gabriel Dominguez Castillo «principal», Edith J. Cisneros } \\
\text { Cohernour «igual» }\end{array}$ \\
\hline $\begin{array}{l}\text { Escritura - Preparación del } \\
\text { borrador original }\end{array}$ & $\begin{array}{l}\text { Edith J. Cisneros Cohernour «principal» J. Gabriel Dominguez } \\
\text { Castillo «igual». }\end{array}$ \\
\hline $\begin{array}{l}\text { Escritura - Revisión y } \\
\text { edición }\end{array}$ & $\begin{array}{l}\text { Edith J. Cisneros Cohernour «principal» J. Gabriel Dominguez } \\
\text { Castillo «igual». }\end{array}$ \\
\hline Visualización & $\begin{array}{l}\text { Edith J. Cisneros Cohernour «principal» J. Gabriel Dominguez } \\
\text { Castillo «igual» Pedro J. Canto Herrera «apoyo». }\end{array}$ \\
\hline Supervisión & $\begin{array}{l}\text { Edith J. Cisneros Cohenour «principal» J. Gabriel Dominguez } \\
\text { Castillo «igual» }\end{array}$ \\
\hline $\begin{array}{l}\text { Administración de } \\
\text { Proyectos }\end{array}$ & $\begin{array}{l}\text { Edith J. Cisneros Cohenour «principal» J. Gabriel Dominguez } \\
\text { Castillo «igual». }\end{array}$ \\
\hline Adquisición de fondos & Edith J. Cisneros Cohenour \\
\hline
\end{tabular}

